\title{
Caracterización de mieles de obtención artesanal y comerciales producidas en Galicia (NO de España) a partir de su espectro polínico y contenido proteico
}

\author{
Ma Pilar de Sá Otero, Emilia Díaz Losada y Sandra Arnesto Baztán ${ }^{1}$
}

Resumen: de Sá Otero, M. P.; Díaz Losada, E. \& Armesto Baztán, S. 2011. Caracterización de mieles de obtención artesanal y comerciales producidas en Galicia (NO de España) a partir de su espectro polínico y contenido proteico. Bot. Complut. 35: 131-140.

Se han estudiado 49 mieles producidas en el NO de España (Ourense-Galicia), 37 de producción artesanal y 12 comercializadas con etiqueta de calidad "Mel de Galicia". Se determinó su origen floral, mediante el método de análisis polínico porcentual, y su contenido proteico, mediante el método de Bradford. En cuanto a su origen floral, han resultado ser 9 monoflorales de Castanea sativa, 6 de Rubus ulmifolius-t., una monofloral de Cytisus scoparius-t. y las demás milflores con predominancia de castaño (Castanea sativa), zarza (Rubus ulmifolius-t.), xesta-tojo (Cytisus scoparius-t.) o brezo (Erica sp.). Por su riqueza polínica, se han encontrado, en las mieles artesanales, 9, de clase de Maurizio I; 9, de Clase II; 9 , de Clase III; 7, de Clase IV y 3, de Clase V. En las comerciales, 4, de Clase IV y 8, de Clase V. El contenido en proteína de las mieles varió desde $0,8 \mathrm{mg}$ prot./g miel a 5,2 mg prot./g miel. Los valores medios han sido de 1,95, para las de origen artesanal y 3,1, para las comerciales.

Palabras clave: miel, melisopalinología, proteína.

Abstract: de Sá Otero, M. P.; Díaz Losada, E. \& Armesto Baztán, S. 2011. Mellisopallinological characterisation of unifloral honeys from artisan and commercial obtainment produced in Galicia (NW of Spain) from its pollen spectra. Bot. Complut. 35: 131-140.

An analysis was made of 49 honeys produced in NW of Spain (Ourense-Galicia), 37 of homemade production and 12 commercialized with quality label "Mel de Galicia" (Galician Honey). Its flower origin was determinate by the method of percentage pollen analysis and its protein content by Bradford method. They resulted to be 9 chestnut tree (Castanea sativa) and 6 bramble (Rubus ulmifolius-t.) unifloral, one Cytisus scoparius-t. unifloral and the other honeys was multifloral with predominance of chestnut (Castanea sativa), bramble (Rubus ulmifolius-t.), shrubland (Erica sp.) with gorse (Cytisus scoparius-t.) or heather. It was found 9 homemade honeys belonged to the Maurizio's Class I, 9 to the Class II, 9 to the Class III, 7 to the Class IV and 3 belong to the Class V. Among the commercial honeys, 4 belong to the Class IV and 8 to the Class V. The value of protein in the honeys varied from 0.8 to $5.2 \mathrm{mg}$ prot./g honey. The average was $1.95 \mathrm{mg}$ prot./g honey for homemade honeys, and $3.1 \mathrm{mg}$ prot./g honey for commercial ones. This wide variation resulted as homemade like commercial honeys, and in this last it seem even to be higher.

Key words: honey, melisopalinology, protein.

\section{INTRODUCCION}

La miel es un producto biológico muy complejo. Sus características físicas, químicas y organolépticas varían notablemente, como consecuencia de la flora de la zona de origen. Es un producto natural, con alto valor biológico y funcional, que se presenta en el mercado sin apenas procesado industrial. Contribuir al conocimiento del valor nutritivo de las mismas es el objetivo principal de este trabajo. Desde hace tiempo se persigue la búsqueda de un método físico-químico que, a través del análisis de distintos parámetros, pudiese conducir a conocer su origen botánico. Entre estos se han considerado parámetros físico-químicos comunes en mieles de distintos orígenes florales como conductividad, cenizas, acidez libre, prolina y pH (Krauze \& Zalewsk 1991). El contenido en aminoácidos ha sido usado con fines de caracterización botánica (Pérez-Arquillue \& Herrera 1987, Lombard et al. 1984 y Conte et al. 1998).

El contenido en proteínas de la miel es una consecuencia directa del contenido polínico de la misma, tanto de su riqueza polínica como de la diversidad de pólenes contenidos en ellas (Crane 1990). Algunos autores como Gheldof et al. (2002) han establecido una relación de propor-

\footnotetext{
${ }^{1}$ Facultad de Ciencias de Ourense, Universidad de Vigo, E-32004 Ourense. saa@vigo.es

Recibido: 20 diciembre 2010. Aceptado: 3 marzo 2011
} 
cionalidad positiva entre la capacidad antioxidante de las mieles y su contenido en proteínas y compuestos fenólicos. Fonseca et al. (1993) han obtenido diferentes contenidos de proteínas en mieles de diferente origen floral. Así, en mieles de azahar hallan valores de 0,85-2,62 g prot/100 g miel, cuyo valor medio es de $1,4 \mathrm{~g}$ prot/ $100 \mathrm{~g}$ miel. En las mieles de eucalipto el rango se establece de $1,0-2,26 \mathrm{~g}$ prot/100 g miel, su valor medio es de $1,84 \mathrm{~g}$ prot/100 g miel. Estos autores establecen además que la cantidad relativa de proteína varía en relación a la naturaleza del polen dominante, y también en función de la naturaleza de las especies encontradas en el espectro en proporciones menores. Otras mieles, de origen floral diverso, también analizadas por éstos, han dado valores cuyo máximo es de 3,2 g prot/100 g miel. Baroni et al. (2002) relacionaron el origen monofloral de las mieles y la presencia de proteínas específicas en las mismas. Padovan et al. (2003) han utilizado la presencia de proteínas en la miel como fuente de carbono para establecer la relación de $\mathrm{C}^{13} / \mathrm{C}^{12}$, como método de detección de fraude en el de origen botánico en las mismas. Azedero et al. (2003) establecen también relaciones directas entre el origen floral de las mieles y su contenido proteico. Por otra parte, Cremonez et al. (1998) consideran el polen como fuente de proteínas en la colmena y logran establecer una relación directa entre la proteína contenida en la hemolinfa de las abejas y el aporte proteico que entra en la colmena, a través del análisis del polen corbicular traído por las abejas a la colmena.

De Sá-Otero et al. (2009) realizaron un análisis del contenido en proteínas de las cargas de polen producido por las abejas en una colmena situada en Viana do Bolo (Ourense, NO de España). El polen de las especies de plantas que alcanzaron un porcentaje relativamente alto en las muestras totales son los que también poseyeron mayor contenido de proteína. Estos fueron: el de Cytisus scopariust., Poáceas silvestres, Quercus robur-t., Sanguisorba minor Scop., Salix fragilis L. and Spergularia rubra-t. En este estudio se hace una comparativa del espectro polínico de las diferentes mieles artesanales y comerciales. Se determina el contenido en proteínas de las mieles estudiadas y la posible relación de éste con su procedencia floral y con la riqueza polínica de las mieles.

\section{MATERIAL Y MÉTODOS}

Análisis polínico porcentual. Se hizo análisis polínico cuantitativo y cualitativo de 49 muestras de miel procedentes de 37 colmenas artesanales en la comarca geopolítica de Allariz-
Maceda y 12 mieles comerciales con la etiqueta de calidad "Mel de Galicia" (Anexo 1). Las muestras artesanales proceden de una única extracción anual correspondiente al mes de agosto de 2000. La extracción se ha llevado a cabo por centrifugación (Fig. 1). La metodología utilizada ha sido la propuesta por Loveaux et al. (1978), partiendo de 20 gramos de cada una de las mieles. Para el análisis porcentual se ha procedido según la metodología propuesta por de Sá-Otero et al. (1993), leyendo cuatro preparaciones microscópicas por muestra de miel. En cada una de ellas se identificaron la cuarta parte del total de pólenes a partir de los que se hizo el cálculo de porcentajes. Las preparaciones microscópicas se han confeccionado sobre glicerina fenolada, sin tinción. Como material de referencia para la identificación polínica, se han utilizado preparaciones microscópicas, elaboradas a partir del polen de plantas recogidas en los alrededores de los colmenares artesanales, la palinoteca existente en el laboratorio de Palinología Aplicada (Melisopalinología y Viticultura) de la Facultad de Ciencias de Ourense y los tratados de Palinología (Moore et al. 1991, Valdés et al. 1986, de SáOtero et al. 1996, Ramil Rego et al. 1992).

Los resultados han sido expresados en las cinco categorías establecidas por Louveaux et al. (1978): polen dominante (D: $>45 \%$ ); polen acompañante (A: 16-45\%); polen importante (I: $3-15 \%)$; polen aislado, raro (m: $1-3 \%$ ) y polen presente (p: $<1 \%$ ). Las identificaciones polínicas se han hecho a nivel de especie, siempre que ha sido posible, familia, género o tipo polínico. El tipo polínico incluye especies y/o géneros presentes en una misma área geográfica que poseen la misma forma polínica. Para el análisis cuantitativo se ha empleado un Neubauer Conting Cell. Los resultados se expresan en forma de clases de frecuencia Maurizio (1939): Clase I, <2 000 granos de polen/g miel; Clase II, entre $2000-10000$; Clase III, entre 10 000-50 000; Clase IV, entre 50 000-100 000 y Clase V > 100000.

Determinación de proteínas. Para la preparación del reactivo Bradford (1976), se pesan $100 \mathrm{mg}$ de Coomasie G-250 y se enrasa a un volumen de $50 \mathrm{~mL}$, con etanol al $95 \%$. A esta solución se le añade ácido ortofosfórico, enrasándola hasta $100 \mathrm{~mL}$. Se agita hasta su completa disolución y finalmente se le añade agua destilada hasta un volumen final de $1.000 \mathrm{~mL}$. Se reserva en la oscuridad hasta su posterior utilización. Para la preparación de la proteína de referencia (seroalbúmina bovina) se pesan $10 \mathrm{mg}$ de seroalbúmina bovina y se disuelven en $10 \mathrm{~mL}$ de agua destilada. La disolución final se divide en alícuotas de $0,5 \mathrm{~mL}$ y se reservan en el congelador. Para la preparación de la muestra problema se disuelven $2,5 \mathrm{~g}$ de miel en $50 \mathrm{~mL}$ de agua destilada. A continuación y en agitación continua, se procede a elevar su $\mathrm{pH}$ hasta 8,5, con $\mathrm{NaOH}$. La recta de calibrado se obtiene a partir de una serie de disoluciones de la proteína patrón a distintas concentraciones, todas ellas con un volumen final de $100 \mu$ l. A continuación se añaden $2 \mathrm{~mL}$ del reactivo Bradford a cada disolución, con el fin de que éste no sea el reactivo limitante en el proceso de unión tinte-proteína. Cada una de estas cubetas reflejará una absorción de luz diferente y proporcional a la concentración de proteína disuelta, lo que proporcionará una recta patrón a la que referir los datos de las muestras problema. La absorbancia se mide a una longitud de onda de $595 \mathrm{~nm}$. 


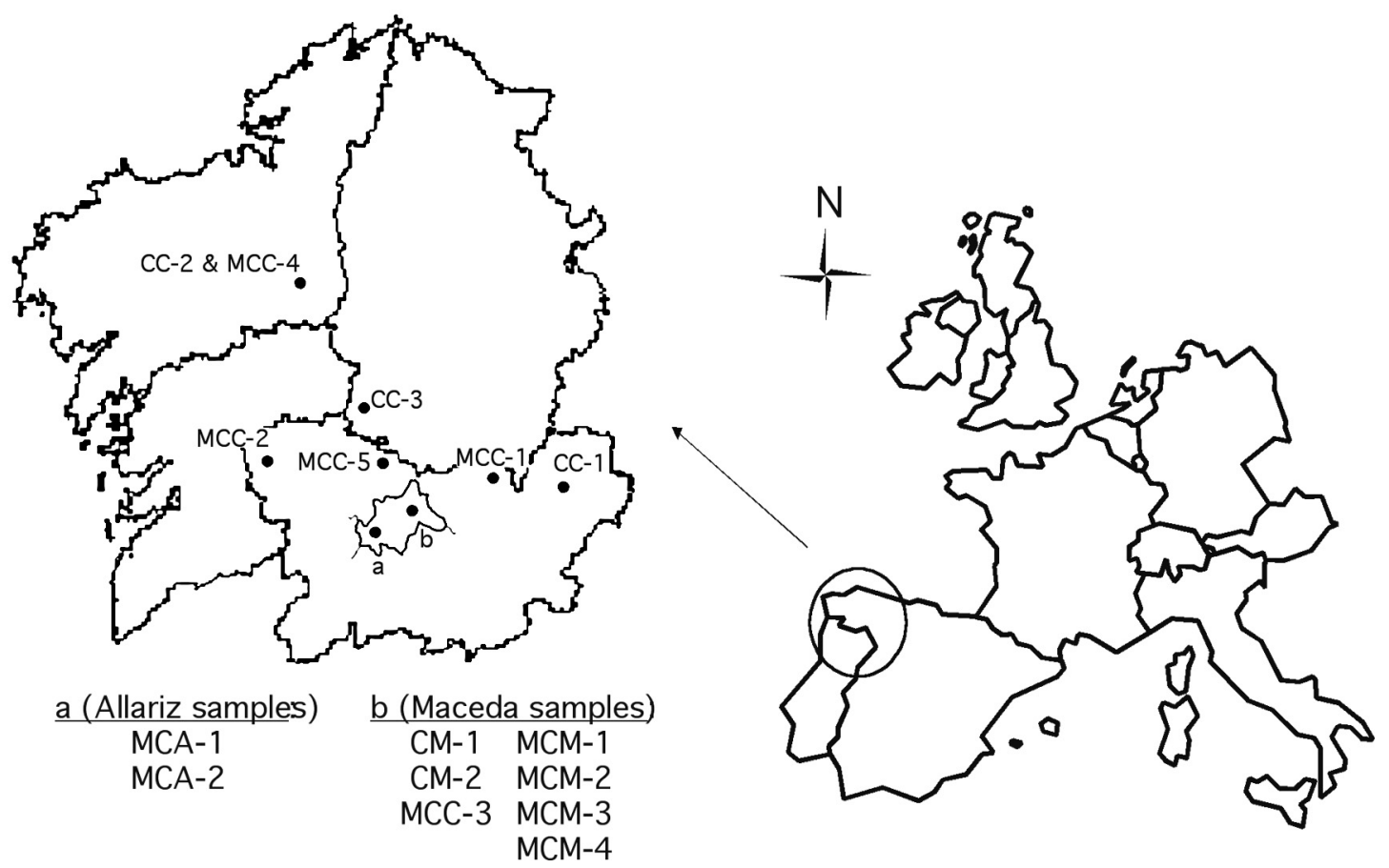

Fig. 1- Situación de las colmenas estudiadas.

La cuantificación de la proteína presente en la muestra problema se lleva a cabo preparando disoluciones de la muestra problema de 40 y $80 \mu \mathrm{L}$, enrasando a $100 \mu \mathrm{L}$, con $\mathrm{H}_{2} \mathrm{O}$ destilada. A cada disolución se le añaden $2 \mathrm{~mL}$ del reactivo Bradford y se procede a leer la absorbancia a $595 \mathrm{~nm}$. Este proceso se realiza por triplicado. El valor medio de la absorbancia obtenido para las tres réplicas permite, en relación a la recta patrón, la determinación de la proteína presente en la muestra problema.

\section{RESULTADOS}

Las mieles han resultado ser monoflorales de castaño (Castanea sativa), 9; monoflorales de zarza (Rubus ulmifolius-t.), 6; monoflorales de Cytisus scoparius-t., 1, y las demás milflores con predominancia de castaño (Castanea sativa), zarza (Rubus ulmifolius-t.), xesta-tojo (Cytisus scoparius-t.) o brezo (Erica sp.). Se han clasificado según Maurizio (1939); 9, de Clase I; 9, de Clase II; 9, de Clase III; 11, de Clase IV y 11, de Clase V (Tabla 1a y 1b). El valor de proteína en las mieles varió desde $0,8 \mathrm{mg}$ prot./g miel a 5,24 mg prot./g miel (Tabla 2), siendo el valor medio de $2,2 \mathrm{mg}$ prot./g miel, con una varianza de 1,1. Hay una elevada variabilidad en los valores de riqueza proteica, tanto en mieles artesanales (de 0,8 a $4,33 \mathrm{mg}$ prot./g miel), como en mieles comerciales (1,30 a 5,24 mg prot./g miel). Estas últimas, en general, alcanzan valores más altos.
En las mieles monoflorales de Castanea sativa los valores de riqueza proteica varían desde un mínimo de 1,7 $\mathrm{mg}$ prot./g miel a 5,1 mg prot./g miel, con un valor medio de 3,9 mg prot./g miel (Tabla 3). En estas mieles los pólenes con potrcenajes más elevados en su espectro polínico son: Castanea sativa, Rubus ulmifolius-t. y Cytisus scoparius-t. (Tablas la y $1 b$ ). En las mieles monoflorales de Rubus ulmifolius-t. el contenido proteico varía de 1,3 a 2,3 $\mathrm{mg}$ prot./g miel, siendo su media de $1,9 \mathrm{mg}$ prot./g miel (Tabla 3). En estas mieles los pólenes con porcentajes más elevados en su espectro polínico son: Rubus ulmifolius-t., Castanea sativa, Cytisus scoparius-t., Echium plantagineum y Frangula alnus (Tablas 1a y 1b). En la miel monofloral de Cytisus scoparius-t. la riqueza proteica es 2,5 mg prot./g miel (Tabla 3). En esta miel los pólenes con porcentajes más elevados en su espectro polínico son: Castanea sativa y Erica sp.

En las mieles milflores, la mayoría llevan como polen más abundante el de Rubus ulmifolius-t. (17 mieles) el contenido en proteína varía de 0,8 a 5,2 mg prot./g miel, con un valor medio de $1,9 \mathrm{mg}$ prot./g miel. Se encontraron dos mieles milflores con Erica sp, como taxón mas abundante, una artesanal (Muestra 13) y una comercial (Muestra 49), Clase III y IV, respectivamente (Tabla 3). Su contenido en proteínas ha sido 3,8 (13) y 5,2 (49) $\mathrm{mg}$ prot./g miel (Tabla 2). Una miel milflores resultó llevar el polen de 


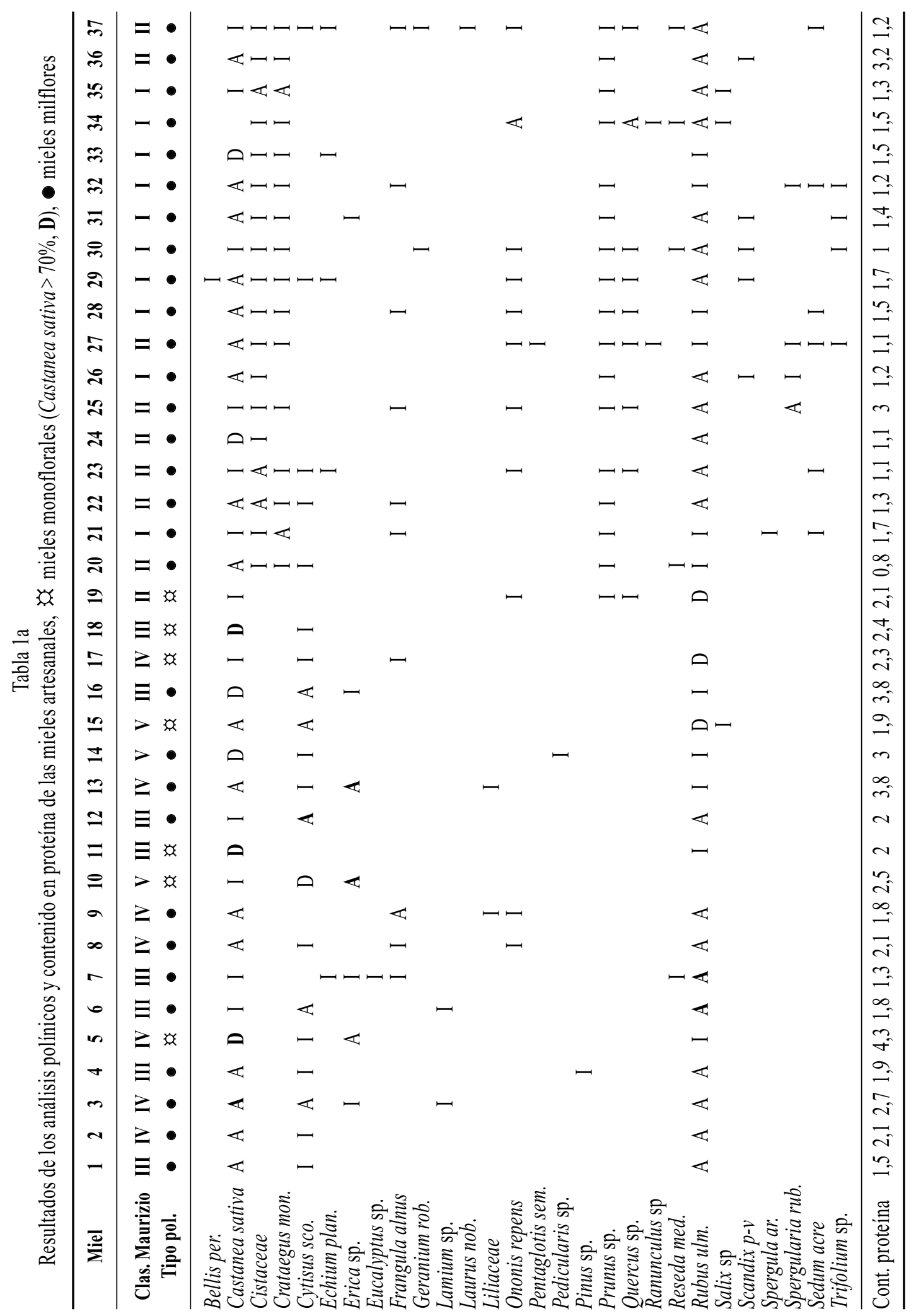


Tabla $1 b$

Resultados de los análisis polínicos y contenido en proteína de las mieles comerciales, mieles monoflorales (Castanea sativa $>70 \%, \mathbf{D})$, $\bullet$ mieles milflores

\begin{tabular}{|c|c|c|c|c|c|c|c|c|c|c|c|c|}
\hline Mieles & 38 & 39 & 40 & 41 & 42 & 43 & 44 & 45 & 46 & 47 & 48 & 49 \\
\hline Clas. Maurizio & V & V & IV & IV & V & V & V & V & V & IV & V & IV \\
\hline Categoría & $x$ & 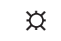 & 议 & 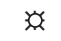 & 好 & 次 & 好 & 娧 & 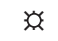 & $\bullet$ & - & - \\
\hline
\end{tabular}

\section{Tipos polínicos}

\begin{tabular}{|c|c|c|c|c|c|c|c|c|c|c|c|c|}
\hline Castanea sativa & A & $\mathrm{A}$ & A & $\mathrm{D}$ & $\mathrm{D}$ & $\mathrm{D}$ & $\mathrm{D}$ & $\mathrm{D}$ & $\mathrm{D}$ & A & $\mathrm{A}$ & A \\
\hline Cytisus scoparius & I & & I & I & & & I & I & A & & A & $\mathrm{A}$ \\
\hline Echium plantagineum & I & & & & & & & & & & & \\
\hline $\begin{array}{l}\text { Erica sp. } \\
\text { Raphanus raphanistrum }\end{array}$ & & & I & & & & & I & & I & I & A \\
\hline $\begin{array}{l}\text { Reseda media } \\
\text { Rubus ulmifolius }\end{array}$ & $\mathrm{D}$ & $\mathrm{D}$ & D & I & I & A & A & $\mathrm{I}$ & I & A & A & \\
\hline Contenido en proteína & 1,8 & 1,3 & 2,3 & 2,8 & 5,1 & 5,1 & 1,7 & 5,1 & 2.5 & 1,4 & 3 & 5,2 \\
\hline
\end{tabular}

Tabla 2

Contenido en proteínas de las mieles

\begin{tabular}{|c|c|c|c|c|c|}
\hline \multicolumn{4}{|c|}{ Mieles artesanales } & \multicolumn{2}{|c|}{ Mieles comerciales } \\
\hline $\mathrm{N} .{ }^{0}$ muestra & $\begin{array}{l}\text { Contenido } \\
\text { en proteína }\end{array}$ & N. ${ }^{\circ}$ de muestra & $\begin{array}{l}\text { Contenido } \\
\text { en proteína }\end{array}$ & $\mathrm{N} .{ }^{0}$ de muestra & $\begin{array}{l}\text { Contenido } \\
\text { en poteína }\end{array}$ \\
\hline 1 & 1,5 & 20 & 0,8 & 38 & 1,8 \\
\hline 2 & 21 & 21 & 17 & 39 & 13 \\
\hline 3 & 2.7 & 22 & 1.3 & 40 & 2.3 \\
\hline 4 & 1,9 & 23 & 1,1 & 41 & 2.8 \\
\hline 5 & 4,3 & 24 & 1,1 & 42 & 5,1 \\
\hline 6 & 1,8 & 25 & 3 & 43 & 5,1 \\
\hline 7 & 2,3 & 26 & 1,2 & 44 & 1,7 \\
\hline 8 & 2,1 & 27 & 1,1 & 45 & 5,1 \\
\hline 9 & 1,8 & 28 & 1,5 & 46 & 2,5 \\
\hline 10 & 2,5 & 29 & 1,7 & 47 & 1,4 \\
\hline 11 & 2,0 & 30 & 1 & 48 & 3,0 \\
\hline 12 & 2,0 & 31 & 1,4 & 49 & 5,2 \\
\hline 13 & 3,8 & 32 & 1,2 & & \\
\hline 14 & 2,0 & 33 & 1,5 & & \\
\hline 15 & 1,9 & 34 & 1,5 & & \\
\hline 16 & 3,8 & 35 & 1,3 & & \\
\hline 17 & 2,3 & 36 & 3,2 & & \\
\hline 18 & 2,4 & 37 & 1,2 & & \\
\hline 19 & 2,1 & & & & \\
\hline \multicolumn{2}{|c|}{ Media } & \multicolumn{2}{|c|}{1,95} & Media & 3,09 \\
\hline \multicolumn{2}{|c|}{ Varianza } & \multicolumn{2}{|c|}{0,67} & Varianza & 2,26 \\
\hline \multirow{2}{*}{\multicolumn{2}{|c|}{ TOTAL MIELES }} & \multirow{2}{*}{\multicolumn{2}{|c|}{$\begin{array}{c}\text { Media } \\
\text { Varianza }\end{array}$}} & \multicolumn{2}{|c|}{2,23} \\
\hline & & & & & \\
\hline
\end{tabular}


Tabla 3

Caracterización de las mieles por su origen floral

\begin{tabular}{|c|c|c|c|c|c|}
\hline \multirow{2}{*}{ Tipo de miel } & \multicolumn{3}{|c|}{ Mieles } & \multirow{2}{*}{$\begin{array}{c}\text { Contenido } \\
\text { proteico (mg/g) }\end{array}$} & \multirow{2}{*}{$\begin{array}{l}\text { C.P. Medio } \\
\text { (mg/g) }\end{array}$} \\
\hline & N. ${ }^{0}$ & Muestra & Cl. Maurizio & & \\
\hline \multicolumn{6}{|l|}{ Monofloral } \\
\hline Castanea sativa $>70 \%$ & 9 & $\begin{array}{r}5 \\
11 \\
18 \\
41 \\
42 \\
43 \\
44 \\
45 \\
46\end{array}$ & $\begin{array}{c}\text { III } \\
\text { V } \\
\text { IV } \\
\text { IV } \\
\text { V } \\
\text { V } \\
\text { V } \\
\text { V } \\
\text { V }\end{array}$ & $\begin{array}{l}4,3 \\
2 \\
2,4 \\
2,8 \\
5,1 \\
5,1 \\
1,7 \\
5,1 \\
2,5\end{array}$ & 3,3 \\
\hline Rubus ulmifolius- $\mathrm{t}>45 \%$ & 6 & $\begin{array}{l}15 \\
17 \\
19 \\
38 \\
39 \\
40\end{array}$ & $\begin{array}{c}\text { V } \\
\text { III } \\
\text { III } \\
\text { V } \\
\text { V } \\
\text { IV }\end{array}$ & $\begin{array}{l}1,9 \\
2,3 \\
2,1 \\
1,8 \\
1,3 \\
2,3\end{array}$ & 1,9 \\
\hline Cytisus scoparius-t. $>45 \%$ & 1 & 10 & IV & 2,5 & 2,5 \\
\hline \multicolumn{6}{|l|}{ Milflores varias } \\
\hline Castanea mayoritario & 11 & $\begin{array}{l}14 \\
16 \\
20 \\
22 \\
24 \\
27 \\
28 \\
31 \\
32 \\
33 \\
36\end{array}$ & $\begin{array}{c}\text { IV } \\
\text { V } \\
\text { II } \\
\text { I } \\
\text { II } \\
\text { I } \\
\text { II } \\
\text { I } \\
\text { I } \\
\text { I } \\
\text { II }\end{array}$ & $\begin{array}{l}3 \\
3,8 \\
0,8 \\
1,3 \\
1,1 \\
1,1 \\
1,5 \\
1,4 \\
1,2 \\
1,5 \\
3,2\end{array}$ & 1,8 \\
\hline Rubus mayoritario & 17 & $\begin{array}{r}1 \\
2 \\
3 \\
4 \\
6 \\
7 \\
8 \\
9 \\
23 \\
25 \\
26 \\
29\end{array}$ & $\begin{array}{l}\text { III } \\
\text { III } \\
\text { IV } \\
\text { IV } \\
\text { IV } \\
\text { III } \\
\text { III } \\
\text { IV } \\
\text { II } \\
\text { II } \\
\text { II } \\
\text { I }\end{array}$ & $\begin{array}{l}1,5 \\
2,1 \\
2,1 \\
1,9 \\
1,8 \\
1,3 \\
2,1 \\
1,8 \\
1,1 \\
3 \\
1,2 \\
1,7\end{array}$ & 1,6 \\
\hline
\end{tabular}


Tabla 3 (cont.)

Caracterización de las mieles por su origen floral

\begin{tabular}{|c|c|c|c|c|c|}
\hline \multirow{2}{*}{ Tipo de miel } & \multicolumn{3}{|c|}{ Mieles } & \multirow{2}{*}{$\begin{array}{c}\text { Contenido } \\
\text { proteico }(\mathrm{mg} / \mathrm{g})\end{array}$} & \multirow{2}{*}{$\begin{array}{l}\text { C.P. Medio } \\
\text { (mg/g) }\end{array}$} \\
\hline & N. ${ }^{0}$ & Muestra & Cl. Maurizio & & \\
\hline & & 30 & I & 1 & \\
\hline & & 35 & I & 1,3 & \\
\hline & & 37 & II & 1,2 & \\
\hline & & 47 & IV & 1,4 & \\
\hline & & 48 & $\mathrm{~V}$ & 3 & \\
\hline \multirow{2}{*}{ Erica mayoritario } & 2 & 13 & III & 3,8 & 4,5 \\
\hline & & 49 & IV & 5,2 & \\
\hline Crataegus mayoritario & 1 & 21 & II & 1,7 & 1,7 \\
\hline Cytisus mayoritario & 1 & 12 & III & 2 & 2 \\
\hline \multicolumn{3}{|c|}{ Mielada (Q. robur-t mayoritario, $987 \mathrm{~g} \mathrm{p} / \mathrm{g}$ miel) 34} & I & 1,5 & 1,5 \\
\hline
\end{tabular}

Crataegus monogyna-t. como taxón mas abundante (Muestra 21), Clase II. Su contenido en proteínas ha sido 1,7 (Tabla 3). Otra miel milflores resultó con polen de Quercus robur-t. como taxón mas abundante (Muestra 34), Clase I (987 g polen/g miel), que hemos considerado como de mielada. Su contenido en proteínas ha sido 1,5 (Tabla 3).

\section{DISCUSIÓN}

Fonseca et al. (1993) establecen que la cantidad relativa de proteína en las mieles varía en relación a la naturaleza del polen dominante, y también en función de la naturaleza de las especies encontradas en el espectro en proporciones menores. Es posible que una parte de las proteínas enzimáticas pueda ser incorporada por la abeja. El hecho de la existencia de mieles carentes de prótidos, como las de acacia, las de cítricos y las de tilo, permiten afirmar que es el contenido en polen el que contribuye mayoritariamente a la presencia de este componente de la miel Crane (1990).

Tabla 4

Absorción de inercia para cada uno de los ejes principales

\begin{tabular}{ccccc}
\hline \multirow{2}{*}{$\begin{array}{c}\text { Porcentaje } \\
\text { de varianza explicado }\end{array}$} & \multicolumn{4}{c}{ Componentes } \\
\cline { 2 - 5 } & I & II & III & IV \\
\hline $64,95 \%$ & 31,94 & 13,22 & 10,28 & 9,47 \\
\hline
\end{tabular}

El $62 \%$ de las mieles muestran valores inferiores a 1,5 $\mathrm{mg}$ prot./g miel, su valor medio es de $2,8 \mathrm{mg}$ prot./g miel y varía de 0,8 a 3,2 mg prot./g miel. Si consideramos los valores medios de las muestras en cada una de las clases de frecuencia de riqueza polínica de Maurizio, prescindiendo de los "datos atípicos" (outhiers), puede observarse que el valor medio de la riqueza proteica en mieles de Clase $\mathrm{V}$ es progresivamente superior al de las mieles Clase I, II, III y IV (Tabla 6). Esta misma relación fue establecida también con anterioridad por Sande González (2004). Las mieles monoflorales de Castanea sativa po-

Tabla 5

Las variables más influyentes en el total de inercia de cada eje en la matriz de componentes principales

\begin{tabular}{lrrrr}
\hline & \multicolumn{4}{c}{ Componentes } \\
\cline { 2 - 5 } & \multicolumn{1}{c}{ I } & \multicolumn{1}{c}{ II } & \multicolumn{1}{c}{ III } & IV \\
\hline Riqueza polínica & 0,889 & $-0,137$ & 0,037 & 0,057 \\
Monofloralidad & 0,771 & 0,061 & 0,029 & 0,244 \\
Cytisus scoparius-t & 0,509 & $-0,165$ & $-0,455$ & $-0,418$ \\
Castanea sativa & 0,634 & 0,473 & 0,220 & 0,297 \\
Quercus robur-t & $-0,562$ & 0,033 & $-0,178$ & $-0,071$ \\
Salix-t & $-0,313$ & 0,082 & $-0,372$ & 0,697 \\
Spergularia rubra-t. & $-0,395$ & 0,374 & 0,273 & 0,177 \\
Ranunculus repens & $-0,225$ & 0,546 & 0,512 & $-0,293$ \\
Raphanus raphanistrum-t. & 0,095 & $-0,449$ & 0,591 & 0,170 \\
Rubus ulmifolius-t & $-0,141$ & $-0,735$ & 0,229 & 0,280 \\
Prunus spinosa-t. & $-0,867$ & 0,112 & $-0,169$ & 0,187 \\
Riqueza proteica & 0,623 & 0,338 & $-0,202$ & 0,247 \\
\hline
\end{tabular}


Tabla 6

Contenido proteico medio de las muestras de miel en cada una de las clases de frecuencia de riqueza polínica de Maurizio, prescindiendo de los valores atípicos (outliers)

\begin{tabular}{|c|c|c|c|c|}
\hline Cl. Mauricio & Media & Varianza & «Valores atípicos» & Rango valores \\
\hline I & 1,3 & 0,047 & & $1-1,7$ \\
\hline II & 1,3 & 0,06 & $\begin{array}{l}20(0,8 \mathrm{mg} \text { prot, } / \mathrm{g} \text { miel }) \\
\text { y } 25(3 \mathrm{mg} \text { prot }, \mathrm{g} \text { miel })\end{array}$ & $1,1-1,7$ \\
\hline III & 2,2 & 0,07 & $\begin{array}{c}5(4,33 \mathrm{mg} \text { prot }, \mathrm{g} \text { miel }) \\
\text { y } 13(3,77 \mathrm{mg} \text { prot,/g miel })\end{array}$ & $1,5-2,3$ \\
\hline IV & 2,2 & 0,15 & $\begin{array}{l}28(1,39 \mathrm{mg} \mathrm{prot}, / \mathrm{g} \text { miel }) \\
\text { y } 30(5,24 \mathrm{mg} \text { prot,/g miel })\end{array}$ & $1,9-2,8$ \\
\hline V & 3,2 & 1,9 & 20 (1,30 mg prot,/g miel) & $1,9-5,1$ \\
\hline
\end{tabular}

seen un valor medio mayor de riqueza proteica que las monoflorales de Rubus ulmifolius-t. (Tabla 3) y su riqueza polínica es semejante. Las mieles milflores muestran variabilidad entre si, y la mayoría de aquellas que incorporan en sus combinaciones polínicas alto porcentaje de polen de Castanea sativa son a su vez más ricas en proteína.

Independientemente de que las mieles sean comerciales 0 artesanales, los valores más frecuentes de proteína se hallan en el rango de 1,5-2,5 mg prot./g miel (29 de 37 artesanales y 7 de 12 comerciales). El 66,7\% de las mieles artesanales tiene valores menores de $2,40 \mathrm{mg}$ prot $/ \mathrm{g}$ miel y el 33,33\% alcanza valores superiores a la media. En las mieles comerciales el $66,67 \%$ posee valores inferiores a 3,09 $\mathrm{mg} \mathrm{prot} / \mathrm{g}$ miel y el 33,33\% valores superiores a la media.

De Sá-Otero et al. (2009) han estudiado el contenido proteico de cada uno de los tipos polínicos mas frecuentes y abundantes en el polen apícola del NO de España, resultando, en orden decreciente, Cytisus scoparius-t., Poáceas silvestres, Castanea sativa, Quercus robur-t., Sanguisorba minor, Salix sp., Spergularia rubra-t., Ranunculus sp., Halimium alysoides, Rhaphanus raphanistrum-t., Rubus ulmifolius-t., Prunus spinosa-t., Plantago lanceolata, Jasione montana, Erica sp.

Para cuantificar como contribuyen a la riqueza proteica de una miel su condición de monofloral o milflores, riqueza polínica y la potencialidad de aporte proteico por parte del polen de las diferentes especies de plantas que componen su espectro, se ha realizado un Análisis de Correspondencias Categorizado (Análisis de Componentes Principales). Para ello se ha construido una matriz en la que se expresan los valores de proteína alcanzados por cada una de las mieles, con sus valores reales; monofloral,
1, milflores 0; riqueza polínica clase I y II, 0; Clase III, 1; Clase IV, 2 y Clase V, 3. Para los diferentes taxones de riqueza proteica conocida se ha establecido en base a su valor de $\%$ alcanzado en las diferentes mieles, si $\%<3,1$; de 3-15\%, 2; 15-45\%, 3 y > 45\%, 4 (de Sá-Otero et al. 2009).

La absorción de inercia para cada uno de los cuatro componentes principales obtenidos (Tabla 4), junto con las variables influyentes, para cada uno de los análisis, se reúne en la Tabla 5. Las variables que contribuyen más intensamente a la inercia total de cada componente se relacionan en la Tabla 5. En relación con la componente uno, la riqueza polínica, la monofloralidad y el porcentaje de Castanea sativa, parecen ser las variables que más contribuyen positivamente a la caracterización de las mieles en relación a su contenido proteico. Los valores negativos alcanzados por Quercus robur-t., Prunus spinosa-t. etc. podrían ser debidos a que las mieles, en las que muestran estos tipos polínicos alcanzan valores mayores a $3 \%$, son de Clase I y II de Maurizio. Para las otras tres componentes parecen contribuir más los valores negativos.

Existe un alto grado de significación en las mieles entre la riqueza polínica, el concepto de monofloralidad y los valores de porcentaje de Castanea sativa, Cytisus scoparius-t., Salix sp., Spergularia rubra-t. y Prunus spino$s a-$ t.

\section{CONCLUSIONES}

De los resultados, a pesar de la escasez de datos publicados de constatación (Azeredo et al. 2003, White \& Rudyj 1978, Deug Chan Lee et al. 1998), se han podido 
extraer las siguientes consideraciones, por el momento de interpretación relativa, en espera de nuevas aportaciones futuras. Las mieles artesanales son de riqueza polínica $\left(\mathrm{n}^{0}\right.$ granos polen/g miel) de clases I, II, III, IV y V de Maurizio (1939). Las mieles comerciales son de Clase IV y V. Parece existir, en términos de generalidad, una relación de proporcionalidad entre la riqueza polínica y el contenido en proteína. Es también posible establecer una relación de proporcionalidad entre la riqueza proteica y la condición de monofloralidad de las mieles, tanto artesanales como comerciales. Las mieles con menor número de granos de polen por gramo de miel, Clase I y II son todas ellas de producción artesanal. Los valores máximos en proteína los han alcanzado mayor número de mieles, en las que son altos los porcentajes de polen de Castanea sativa.

\section{AGRADECIMIENTOS}

Las autoras quieren agradecer a Cristina Pardo Martín la revisión de este artículo.

\section{BIBLIOGRAFÍA}

Azedero, L. C.; Azedero, M. A.; De SouzA, S. R. \& Dutra, V. M. L. 2003. Protein contents and physicochemical properties in honey simples of Apis mellifera of different floral origins. Food Chem. 80(2): 249-254.

Baroni, M. V.; Baroni, M. V.; Chiabrando, G. A.; Costa, C.; WUNDERLIN, D. A. 2002. Assessment of the floral origin of honey by SDS-PAGE Inmunoblot Techniques. J. Agric. Food Chem 50: 1362-1367.

BRADFORD, M. M. 1976. A rapid and sensitive method for the quantitation of microgram quantities of protein utilizing the principle of protein dye binding. Anal. Biochem. 72: 248-254.

Conte, L. S.; Miorini, M.; Giomo, A.; Bertacco, G. \& Zironi, R. 1998. Evaluation of some fixed components for unifloral honey characterization. J. Agric. Food Chem. 46: 18441849.

CRANE, E. 1990. Bees and beekeeping. Cornell University Press, New York.

Cremonez, T. M.; Jong, D. \& Bitondi, M. M. G. 1998. Quantification of hemolymph proteins as a fast method for testing protein diets for money bees (Hymenoptera: Apideae) $J$. Econ. Entomol. 91(16): 1284-1289.

De sá-otero M. P; Díaz losada, E. \& González Porto, A. V. 1993. Estudio estadístico de la representatividad de los datos obtenidos en análisis polínicos de mieles de Orense (España). Bol. Soc. Esp. Hist. Nat. (Sec. Biol.) 90(1-4): 5-16.

De sá-otero M. P.; SuÁrez-Cervera, M. \& Gracia, V. R. (Eds.). 1996. Atlas de polen de Galicia. Diputación de Ourense.

De sá-otero M. P.; Armesto-baztán, S. \& Díaz-losada, E. 2009. Analysis of protein content in pollen loads produced in north-west Spain. Grana 48: 290-296.

Deug-Chan-LeE; SAnG-Young-LeE; SANG-HOON-ChA; Yong-SOONCHOI \& HAE-IK-RHEE. 1998. Discrimination of native beehoney and foreign beehoney by SDS-PAGE. Korean J. Food Sci. Technology 30(1): 1-5.

Fonseca E., KleinerT, A. \& Amaral, A. D. 1993. Méis Brasileiros: estudio da sua origen floral. Apicultura no Brasil 16(5): 30-33.
Gheldof, N.; Wang, X. H. \& ENGeSETH, N. J. 2002. Identification and quantification of antioxidant components of honeys from various floral sources. J. Agric. Food Chem. 50: 5870-5877.

Krauze, A. \& Zalewski, R. 1991. Classification of honeys by principal component analysis on the basis of chemical and physical parameters. Eur. Food Res. Technol. 192(1): 19-23.

Lombard A.; Buffa, M.; Manino, A. \& Patetta, A. 1984. Identification of raffinose in honeydew. Experientia 40(2): 178-180.

Louveaux, J.; Mauricio, A. \& Vorwohl, G. 1978. Methof of Melissopalynology. Bee World. 59(4): 139-157.

Maurizio, A. 1939. Untersuchungen zur quantitativen Pollenanalyse des Honings. Mitt. Geb. Lebenmittelunters 30: 27-69.

Moore, P. D., WebbJ, A. \& Collinson, M. E. 1991. Pollen analysis. Ed. Blackwell Science. Oxford.

Padovan, G. J.; De Jong, D.; Rodrigues, L. P. \& Marchini, J. S. 2003. Detection of adulteration of commercial honey samples by the 13C/12C ratio. Food Chem. 82: 633-636.

Pérez-Arquillué C. \& Herrera Marteache, A. 1987. Análisis de aminoácidos proteínicos en mieles de Los Monegros (España). Alimentaria, Junio: 6771.

Ramil Rego, P.; Aira Rodríguez, M. J. \& Sá-Otero, M. P. 1992. Clave polínica de las Ericáceas gallegas. Lazaroa 13: 33-40.

SAnde GonzáLez, L. 2004. Análisis del contenido proteico de mieles florales de producción artesanal y comercial. Proyecto fin de carrera. Facultad de Ciencias de Ourense. Universidad de Vigo.

Valdés B.; Diez, M. J. \& FernándeZ, B. (Eds.). 1987. Atlas polínico de Andalucía Occidental. Instituto de Desarrollo Regional, Universidad de Sevilla, Excma. Diputación de Cádiz. Sevilla.

WhITE, J. W. Jr. \& RuDYJ, O. N. 1978. The protein content of honey. J. Apic. Res. 17: 234-238. 
Anexo I

Muestras de miel artesanal, 1, Outeiro Torre I; 2, Outeiro Torre II; 3, Asadur; 4, Baños de Molgas; 5, C. Escuadro I; 6, X. Espadañado; 7, Cima de Vila; 8, Batán; 9, Parada; 10, Arnuide; 11, C. Escuadro II; 12, Cuatro Caminos; 13, Porto; 14, A Lama; 15, Pardeconde; 16, Vixueses; 17, Sarreaus; 18, Calvelo; 19, Abauin; 20, Allariz; 21, Enfestela; 22, Folgoso; 23, Fondevila; 24, Magarelos; 25, Novás; 26, 0 Orraca; 27,
Queiroás; 28, 0 Rodicio; 29, San Miguel; 30, San Vitorio; 31, Sta. Mariña; 32, Sto. Estevo; 33, Seoane; 34, Torneiros; 35, A Torre; 36, Valverde; 37, V. Flores; 38-49, Mieles comerciales: 38, A-487265; 39, A-321609; 40, A-296132; 41, OR2301663; 42, OR-0001; 43, A-318168; 44, OR-2302153; 45, OR-0002; 46, A-330039; 47, A-487073; 48, A-334187; 49, A221892 